\title{
Consumer's Response to Negative Corporate Social Responsibility Event-The Perspective of Construal Level Theory: An Abstract
}

\author{
Aihwa Chang, Timmy H. Tseng, and Pei-Ju Tung
}

\begin{abstract}
Communicating CSR performance and fostering a better CSR image on consumer's mind is crucial to a company's competiveness in the current era. However, seldom research examines how consumers process CSR information from the perspective of construal level theory (CLT). Based on CLT, this research conducts a 2 (spatial distance) $\times 2$ (social distance) $\times 2$ (thinking style) betweensubject experiment to examine the joint effects of psychological distances of a negative CSR event and consumers' thinking styles (abstract versus concrete) on their CSR image deteriorations toward the company. The results indicate that negative CSR events in both far and near countries equally worsen CSR image. Furthermore, spatial distance and social distance of the negative CSR event interact to influence CSR image deterioration. Less image deterioration due to closer social distance toward the company is more salient when negative CSR events occur in a home country than in a foreign country. Moreover, when consumer's thinking style is more often at an abstract level, the negative CSR event occurring in foreign country, compared to that in home country, results in higher CSR image deterioration. Based on the findings, this study suggests companies avoid negative CSR events even in a foreign country as negative CSR events in spatial distance still worsen the CSR image of the company. In addition, companies can shield the damage of negative CSR events by building better relationships with consumers in home country because consumers with supportive attitudes toward the company tend to generate counterarguments toward negative events when the events occur in a familiar environment. Finally, since CSR image deterioration is high when there is a match between the spatial distance of the negative CSR event and the construal level of consumer's thinking style, companies can lower the impacts of negative CSR event by making it less fit to consumers' construal level. Companies may communicate negative CSR events occurring in remote countries with a more concrete way while in home country with a more abstract way.
\end{abstract}

\footnotetext{
A. Chang $(\bowtie) \bullet$ T.H. Tseng $\bullet$ P.-J. Tung

National Chengchi University, Taipei, Taiwan, Republic of China

e-mail: aihwa@nccu.edu.tw; littlebeeballball@hotmail.com; peiju0601@gmail.com
} 